\title{
Bulletin of the American Mathematical Society
}

This journal is the official organ of the Society. It reports official acts of the Society and the details of its meetings. It contains some of the officially invited addresses presented before the Society, reviews of advanced mathematical books, research problems and a department of research announcements.

The subscription price is $\$ 12.00$ per annual volume of six numbers.

Research Problems and Invited Addresses offered for publication should be sent to MURRAy GerSTENHABER, School of Mathematics, Institute for Advanced Study, Princeton, New Jersey 08540; Book Reviews to FELIX BROWDER, Department of Mathematics, University of Chicago, Chicago, Illinois 60637. Research Announcements offered for publication should be sent to some member of the Council of the Society, and communicated by him to M. H. PROTTER, Department of Mathematics, University of California, Berkeley, California 94720. All other communications to the editors should be sent to the Managing Editor, FELIX BROwDER.

The members of the Council for 1967 are: A. A. Albert, R. D. Anderson, H. A. Antosiewicz, R. F. Arens, Maurice Auslander, P. T. Bateman, R. H. Bing, Felix Browder, William Browder, David Buchsbaum, R. C. Buck, A. P. Calderon, Lamberto Cesari, P. J. Cohen, P. E. Conner, C. W. Curtis, J. B. Diaz, Eldon Dyer, Samuel Eilenberg, Herbert Federer, Walter Feit, Murray Gerstenhaber, J. W. Green, P. R. Halmos, O. G. Harrold, S. Helgason, Henry Helson, M. H. Heins, Kenneth Hoffman, Eugene Isaacson, Norman Levinson, G. W. Mackey, Saunders Mac Lane, W. T. Martin, A. P. Mattuck, H. P. McKean, Jr., C. B. Morrey, J. K. Moser, G. D. Mostow, Louis Nirenberg, Ivan Niven, R. S. Palais, Frank Peterson, R. S. Pierce, Everett Pitcher, M. H. Protter, Irving Reiner, Gian-Carlo Rota, Dana Scott, George Seligman, Stephen Smale, N. E. Steenrod, Michio Suzuki, J. D. Swift, W. R. Wasow, Bertram Yood, John Wermer.

\section{Proceedings of the American Mathematical Society}

This journal is devoted entirely to research in pure and applied mathematics and is devoted principally to the publication of original papers of moderate length. A department called Shorter Notes was established for the purpose of publishing very short papers of an unusually elegant and polished character, for which there is normally no other outlet.

Papers in algebra and number theory should be sent to Arthur P. Matruck, Department of Mathematics, Massachusetts Institute of Technology, Cambridge, Massachusetts or IRVING REINER, Department of Mathematics, University of Illinois, Urbana, Illinois; in probability, real variables, logic, and foundations to R. C. Buck, Department of Mathematics, University of Wisconsin, Madison, Wisconsin 53705; in abstract analysis to either R. C. Buck, or WolfGANG WAsow, Van Vleck Hall, University of Wisconsin, Madison, Wisconsin 53706; in geometry and topology to ELDON DYER, The City University of New York, Graduate Studies Division, 33 West 42nd Street, New York, New York 10036; in other branches of analysis, applied mathematics, and all other fields to MAURICE HeINs, Department of Mathematics, University of Illinois, Urbana, Illinois or WoLFGANG WASOw. All other communications to the editors should be addressed to the Managing Editor, MAURICE HeIns.

\section{Transactions of the American Mathematical Society}

This journal is devoted entirely to research in pure and applied mathematics, and includes in general longer papers than the PrOcEEDINGs.

Papers in analysis and applied mathematics should be sent to Louis NIRENBERG, Courant Institute of Mathematical Sciences, New York University, New York, New York 10012: in topology to FRANKLIN PETERSON, Department of Mathematics, Massachusetts Institute of Technology, Cambridge, Massachusetts 02139; in algebra, number theory, and logic to D. A. BUCHSBAUM, Department of Mathematics, Brandeis University, Waltham, Massachusetts 02154; in geometry and abstract analysis to R. S. PALAIS, Department of Mathematics, Brandeis University, Waltham, Massachusetts 02154; in statistics and probability to H. P. McKeAN, Department of Mathematics, Rockefeller University, New York, New York 10021; in mathematical logic and foundations to DANA ScorT, Department of Mathematics, Stanford University, Stanford, California 94305. All other communications to the editors should be addressed to the Managing Editor, DANA Scort. 


\section{Journals Published by the American Mathematical Society}

\section{Soviet Mathematics-Doklady}

Soviet Mathematics-DokdaDY is a translation journal containing the entire pure mathematics section of the DOKLADY AKADEMI NAUK SSSR, the Reports of the Academy of Sciences of the USSR. The DoKIADY for a year contains about 500 articles, each about 4 pages long. Issued bimonthly.

\section{Mathematical Reviews}

This journal is devoted to abstracts and reviews of the current mathematical literature of the world. Two volumes of MATHEMATICAL REVIEWS will be published in 1967, Volume 33, and Volume 34. Each volume will consist of 6 regular issues plus an index issue. In each regular issue the abstracts and reviews are grouped under subject headings. Publication began in 1940.

\section{Notices of the American Mathematical Society}

This journal announces the programs of the meetings of the Society. It carries the abstracts of all contributed papers presented at the meetings of the Society and publishes news items of interest to mathematical scientists.

All communications should be addressed to the Editor, P.O. Box 6248, Providence, Rhode Island 02904. News items and insertions for each issue must be in the hands of the editor on or before the deadline for the abstracts for the papers to be presented in the meetings announced in that issue. These deadlines are published regularly on the back of the title page.

\section{Mathematics of Computation}

A journal devoted to original papers in numerical analysis, the application of numerical methods and high-speed calculator devices, the computation of mathematical tables, the theory of high-speed calculating devices and other aids to computation. In addition it publishes reviews and notes in these and related fields.

Prospective publications should be addressed to the Editor, Professor Eugene Isaacson, Courant Institute of Mathematical Sciences, New York University, 251 Mercer Street, New York, New York 10012. The author may suggest the name of an editor for review of his paper.

\section{Chinese Mathematics-Acta}

This is a cover-to-cover translation into English of Acta Mathematica Sinica, a bimonthly published by Academia Sinica Peking, People's Republic of China. Acta Mathematica Sinica contains current research in all fields of pure mathematics. 


\section{CONTENTS-Continued from back cover}

Donald G. Babbitt. Wiener integral representations for certain semigroups which have infinitesimal generators with matrix coefficients........................

P. Szeptycki. Some remarks on the extended domain of Fourier transforms........................

Louis Sucheston. On the ergodic theorem for positive operators................................

B. E. Johnson. A commutative semisimple annihilator Banach algebra which is not dual.....................

Neil S. Trudinger. The Dirichlet problem for nonuniformly elliptic equations.........................

M. E. Herrera. De Rham theorems on semianalytic sets...

Gérard Lallement and Mario Petrich. Structure of a class of regular semigroups and rings...................

Stanley J. Osher. Canonical forms of certain Volterra integral operators and a method of solving the commutator equations which involve them.....................

Michael J. Fisher. Singular integrals on Hilbert space......

David Williams. On local time for Markov chains.........

Clifford J. Earle. The contractibility of certain Teichmüller spaces.................................

Frank R. Loscalzo and Thomas D. Talbot. Spline function approximations for solutions of ordinary differential equations.................................

I. Namioka and E. Asplund. A geometric proof of RyllNardzewski's fixed point theorem.................

W. E. Singletary. The equivalence of some general combinatorial decision problems......................

Joachim Weidmann. The virial theorem and its application to the spectral theory of Schrödinger operators..........

O. G. Harrold, Jr. and G. L. Seebeck, III. Locally weakly flat spaces.............................

Marcelle Friedman. Quasi-periodic solutions of nonlinear ordinary differential equations with small damping......

$\mathrm{J}$. de Groot. An isomorphism principle in general topology. .

T. J. Rivlin. Some explicit polynomial approximations in the complex domain.........................

Felix E. Browder. Nonlinear accretive operators in Banach

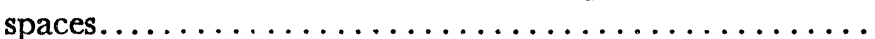

Isaac Chavel. On normal Riemannian homogeneous spaces of rank 1..............................

Bart Braden. Restricted representations of classical Lie algebras of types $A_{2}$ and $B_{2} \ldots \ldots \ldots \ldots \ldots \ldots \ldots \ldots \ldots$

William Stenger. An inequality for the eigenvalues of a class of self-adjoint operators.........................

James Simons. A note on minimal varieties............

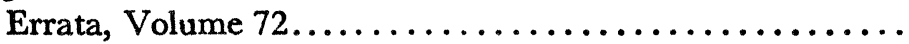




\section{CONTENTS}

May, 1967

\section{Invited Address}

W. H. J. Fuchs. Developments in the classical Nevanlinna theory of meromorphic functions...............

\section{Book Review}

Edwin Hewitt and K. A. Ross. Abstract harmonic analysis.

Reviewed by Leopoldo Nachbin.............

The Annual Meeting in Houston. . . . . . . . . . . . . . . . 295

The February Meeting in New York ............... 306

\section{Research Announcements}

William Parry. Principle partitions and generators. .......

Samir Khabbaz and Everett Pitcher. Critical submanifolds of differentiable mappings. II.................

George J. Minty. On the generalization of a direct method of the calculus of variations. . . . . . . . . . . . . . . .

Felix E. Browder. Existence and perturbation theorems for nonlinear maximal monotone operators in Banach spaces.

O. Shisha and B. Mond. Differences of means . . . . . . . . .

Larry Smith. Multiplicative fibre maps............

H. E. Rauch. The vanishing of a theta constant is a peculiar

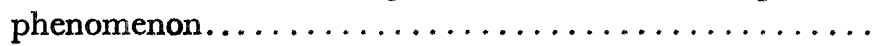

H. E. Rauch. The local ring of the genus three modulus space at Klein's 168 surface. . . . . . . . . . . . . . . .

Shoshichi Kobayashi. Intrinsic metrics on complex mani-

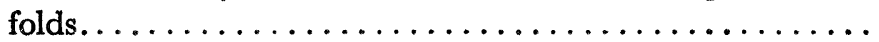

Harry Furstenberg. Poisson boundaries and envelopes of discrete groups. . . . . . . . . . . . . . . . . . .

Robert Kaufman. An example in the calculus of Fourier

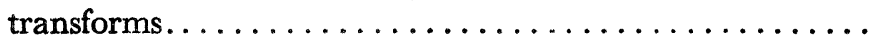

Leonard E. Baum and J. A. Eagon. An inequality with applications to statistical estimation for probabilistic functions of Markov processes and to a model for ecology........

D. G. DeFigueiredo and L. A. Karlovitz. On the radial pro-

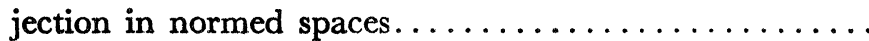

Walter Rudin. Inner functions in polydiscs............

Walter Koppelman The Cauchy integral for functions of several complex variables..................

Ricardo Nirenberg and R. O. Wells, Jr. Holomorphic approximation on real submanifolds of a complex manifold.

John Lamperti. Continuous state branching processes......

David A. Sprecher. On the closure of certain Banach spaces of functions of several variables............. 387

William Margulies. Analytic singular integral operators.... 391 